\title{
Search for Nonradial Pulsations in the Be Star $\omega$ Ori during the MuSiCoS Campaign $1998^{1}$
}

\author{
C. Neiner ${ }^{2}$
}

Observatoire de Paris-Meudon, UMR 8633 CNRS, France \&

Sterrenkundig Instituut Anton Pannekoek, University of Amsterdam

\author{
A.-M. Hubert, M. Floquet \\ Observatoire de Paris-Meudon, UMR 8633 CNRS, France
}

\author{
H.F. Henrichs
}

\section{Sterrenkundig Instituut Anton Pannekoek, University of Amsterdam}

and the MuSiCoS team

\begin{abstract}
Ori is a rapidly rotating B2IIIe star with known spectral variability. We applied a frequency analysis to the MuSiCoS (MUlti SIte COntinuous Spectroscopy) campaign 1998-dataset to search for lineprofile variations (lpv). Variability was found in all studied spectral lines and interpreted as due to non-radial pulsations (NRP).
\end{abstract}

\section{Introduction and observations}

Be stars are nonsupergiant B stars which at least once displayed Balmer emission. They exhibit strongly variable winds and spectral and light variability. The episodic ejection of mass is called the Be phenomenon. Their envelope is flattened by their high rotational velocities, but the centrifugal force by itself cannot explain the presence of a disk. The key problem is then how to eject mass in a nonsteady fashion into a disk.

$\omega$ Ori is a B2IIIe star with $v \sin i=170 \mathrm{~km} \mathrm{~s}^{-1}, T_{\text {eff }}=20240 \mathrm{~K}, \log g=$ $3.48, V_{\mathrm{rad}}=21.8 \mathrm{~km} \mathrm{~s}^{-1}$ and $P_{\text {rot }}=1.46 \mathrm{~d}$ (Zorec, private communication). Stellar wind enhancement and light modulation in optical photospheric and sub-photospheric lines have been detected with a period of about $2 \mathrm{~d}$ (Balona et al., 1992), whereas the stellar wind was modulated at about $1 \mathrm{~d}$ in 1.987 and $1.2 \mathrm{~d}$ in 1996 (Peters \& Gies, 2000). The star entered an active phase 20 years ago. 240 high-S/N spectra of $\omega$ Ori were obtained in 1998 with 8 telescopes (see Henrichs et al., 2000 and Neiner et al., 2001). Several lines were used to search for line profile variations (HeI 4388, 4471, 4713, 4921, 5876, 6678, SiIII 4553,

\footnotetext{
${ }^{1}$ Observations at OHP152, OHP193, Kitt Peak, ESO Heros, Xinglong, Mt Stromlo, LNA, INT

${ }^{2}$ e-mail: Coralie.Neiner@obspm.fr
} 


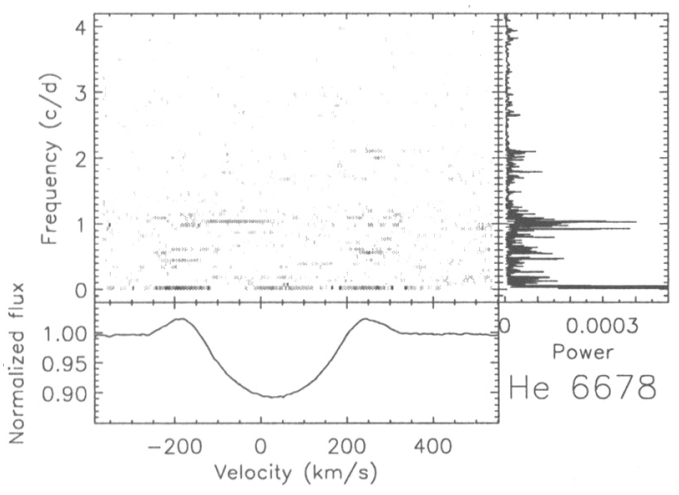

Figure 1. Frequency analysis for HeI 6678: top left: periodogram, bottom: mean profile, right: power spectrum.

CII 4267, MgII 4481 and $\mathrm{H} \alpha$ ) using different methods, especially the Restricted Local Cleanest (Domiciano de Souza Jr, 1999).

\section{Results and conclusions}

All methods clearly show the presence of a significant group of frequencies around $1.03 \mathrm{c} / \mathrm{d}$ in the studied lines, which is incompatible with the $0.68 \mathrm{c} / \mathrm{d}$ rotational frequency. It can definitely be excluded as due to a 1-day alias. From the phase fit we obtained, the NRP mode parameters $|m|=2$ and $\ell=2-3$. The results for the HeI 6678 line are shown here. Note, that a magnetic field could be at work in this star too: our single measurement shows a weak Zeeman signature and is the subject of further study. Another frequency appears around $0.6 \mathrm{~cd}^{-1}$ in the wings of emission lines. This signal does not come from the star itself but from the disk and therefore cannot be interpreted as due to NRPs. It could be associated with the rotational frequency. A mid-term variation of about $22 \mathrm{~d}$ is also detected but the period cannot be precisely determined as the observing run was too short. A study of $\omega$ Ori with a longer database is necessary.

\section{References}

Balona, L.A., Cuypers, J., \& Marang, F. 1992, A\&AS, 92, 533

Domiciano de Souza Jr, A. 1999, PhD Thesis, University of Sao Paulo

Henrichs, H.F., et al. 2000, in ASP Conf. Ser., Vol. 214, The Be phenomenon in Early-Type Stars, eds. M. Smith, H. Henrichs, \& J. Fabregat, (San Francisco ASP), 372

Hubert, A.-M. \& Floquet, M. 1998, A\&A, 335, 565

Neiner, C., et al., 2001, A\&A, in preparation

Peters, G.J. \& Gies, D. 2000, in ASP Conf. Ser., Vol. 214, The Be phenomenon in Early-Type Stars, eds. M. Smith, H. Henrichs, \& J. Fabregat, (San Francisco ASP), 375 